\title{
El huerto escolar como herramienta pedagógica para la asignatura de Ciencia Salud y Medio Ambiente en el Tercer ciclo del Centro Escolar Instituto Nacional de Santa Ana
}

\author{
María Teresa Castaneda \\ Maestra en Asesoría Educativa \\ Docente investigadora, Facultad de Ciencias y Humanidades \\ Universidad Católica de El Salvador \\ maria.castaneda@catolica.edu.sv
}

\section{Resumen}

La enseñanza de las Ciencias Naturales supone un cambio en el modelo didáctico para mejorar los procesos de enseñanza tradicionales y proponer actividades innovadoras, que lleven a la experimentación y al fortalecimiento de la relación de la teoría con la práctica. En respuesta a ello, el objetivo de esta investigación fue incorporar la Metodología del Aprendizaje Basado en Proyectos (ABP), en la utilización del huerto escolar como recurso pedagógico y el desarrollo de prácticas experimentales en esta aula ecológica, dentro de la asignatura de Ciencias, Salud y Medio Ambiente del Tercer ciclo de educación básica.

Los resultados obtenidos fueron satisfactorios, ya que se logró la adquisición de aprendizajes significativos por parte de los estudiantes, construyendo el conocimiento a través de la práctica directa con la naturaleza para la contextualización e integración de los contenidos conceptuales vistos en clase. Se fortalecieron no solo sus competencias científicas, sino también sus habilidades de trabajo en equipo, solidaridad, compromiso y servicio; interactuando con sus compañeros y aplicando la interdisciplinariedad como objeto principal en los procesos del saber hacer y el saber ser en el contexto.

Como producto final se logró la propuesta de una guía metodológica en la que se fundamenta el trabajo en la asignatura de Ciencias, Salud y Medio Ambiente, permitiendo así la integración de materias como: Matemática, Lenguaje, Estudios Sociales e Inglés, lográndose la integración de asignaturas dentro del huerto, dejando un precedente de innovación educativa dentro de un centro escolar complejo, que alberga a una amplia población estudiantil del sistema educativo salvadoreño.

Palabras clave: Huerto escolar, laboratorio en vivo, aula ecológica, Aprendizaje Basado en Proyectos (ABP), Ciencias Naturales, integración de asignaturas.

\begin{abstract}
Teaching Natural Science means a change in the didactic model to increase the traditional learning process in order to promote innovative activities that lead to experimentation and strengthening the relationship between theory and practice. Based on the aforementioned, the objective of this research was to incorporate the Methodology of Project Based Learning (PBL) in the implementation of the school garden as a pedagogical resource and development of experimental practices in the subject of Natural Science in elementary school education.

The results were satisfactory since the acquisition of significant learning by the students was totally achieved, building knowledge through direct practice with nature for the contextualization and integration of the conceptual contents studied in class. In addition, student strengthened not only their scientific skills, but also their skills of teamwork, solidarity, commitment and service; interacting with their peers and applying interdisciplinary interactions as the main object in the process of know-how and knowing how to be in context.

The result was the proposal of a methodological guide in which it is made an emphasis on Natural Science to allow an integration of subjects such as, Mathematics, Language, Social Studies and English. Indeed, it achieved the integration of subjects within the garden, leaving a precedent of educational innovation within a complex school, which houses a large student population of the Educational System of El Salvador.
\end{abstract}

Key Words: School Garden, live laboratory, ecological classroom, Methodology of Project Based Learning (PBL), Natural Sciences, Integration of Subjects. 


\section{Introducción}

Desde la perspectiva de la educación, un proyecto se puede definir como una estrategia de aprendizaje que permite alcanzar uno o varios objetivos (ejemplo: conocimiento de prácticas agrícolas y emprendedurismos), a través de la puesta en práctica de una serie de acciones, interacciones y recursos.

La elaboración de proyectos se transforma en una estrategia didáctica que forma parte de las denominadas metodologías activas, en donde el proyecto se concibe como la búsqueda de una solución inteligente al planteamiento de un problema o una tarea relacionada con el mundo real. El propósito de este es ayudar en la solución de problemas que son complejos y no tienen soluciones sencillas (Blank, 1997; Harwell, 1997; Martí, 2010 citado por Martí, J. A., et. al. 2010).

De acuerdo con Katz y Chard (2000), citado por Gómez y Quintanilla (2015),

(...) el trabajo por proyectos puede ser concebido como una particular forma de realizar investigaciones en la enseñanza de las ciencias. Implica un proceso de investigación de un tema que, en el contexto escolar, vale la pena estudiarse; puede ser realizado por un estudiante, un grupo pequeño de éstos o por todo el grupo que conforma la clase (...)

El trabajo por proyectos en el contexto escolar permite lograr varios objetivos en la enseñanza de las Ciencias Naturales, debido a que se genera un ambiente de aprendizaje que promueve la continua participación de los estudiantes. Además, la puesta en práctica del método científico permite formar en valores y actitudes; así como el trabajo en equipo, la sana convivencia, entre otros.

La estrategia por proyectos conlleva al desarrollo de tres fases que son, según Gómez y Quintanilla (2015, p. 23):

Planeación: secuencia de actividades que deberán desarrollarse, se debe lograr una flexibilización en función de la marcha del proyecto.

Ejecución: Definiendo el planteamiento de la problemática a partir de una pregunta generadora, ejecutando las actividades que permitan establecer un proceso que conlleve a su resolución del problema y a un producto en específico.

Evaluación: Se puede revisar el impacto del trabajo por proyectos en términos de procesos de aprendizaje logrados y de resultados alcanzados, en función de los objetivos propuestos; por lo que es relevante que durante la marcha de las actividades se vaya recogiendo información acerca tanto del proceso como de sus resultados.

\section{Las prácticas de laboratorio en vivo: el huerto como aula ecológica}

El huerto escolar permite al alumnado un aprendizaje que se constituye un laboratorio natural y vivo; de manera que tengan la oportunidad de aplicar experimentalmente, $y$ estableciendo una relación entre la teoría y la 
práctica de modo vivencial al investigar.A su vez, indagar y trabajar como un verdadero científico, poniendo en práctica y/o haciendo ciencia escolar. Esto en palabras de la $\mathrm{FAO}^{1}$ (2009), es tener "la oportunidad de adquirir mayor destreza y técnicas para mejorar su calidad de vida, la de su familia y su comunidad”.

Este recurso es reconocido por facilitar y enriquecer el aprendizaje de los educandos, el cual se constituye en un laboratorio natural y vivo; "de manera que los estudiantes tengan la oportunidad de aplicar experimentalmente y establecer relación entre la teoría y la práctica de un modo vivencial al investigar, indagar y trabajar como un verdadero científico" (Mancilla, 2014, p. 24).

El huerto supone convertir al estudiantado en investigadores y sumergirlos en el trabajo científico, teniendo en cuenta la importancia de crear un trabajo colectivo y permanente para organizar, administrar el mismo huerto y aprender en él. Si bien la educación está avanzando, en algunas instituciones educativas se siguen utilizando los métodos tradicionales para la enseñanza de las Ciencias Naturales; en consecuencia, no se están aprovechando las ventajas con que cuenta el entorno natural mismo de los Centros Educativos como medio de aprendizaje. Por ello, resulta necesario la práctica de un tipo de enseñanza que acerque al estudiante al mundo que le rodea, y que le permita encontrar la explicación científica a interrogantes que este acercamiento plantea.

\section{Metodología}

El estudio tuvo una modalidad de investigación acción, fundamentada en la realidad del alumnado y del contexto; donde el consenso y el trabajo en equipo fueron la clave de la funcionalidad de las actuaciones. Ante esto se buscó un Centro Educativo que reuniera las siguientes características:

- Institución pública que contara con un espacio abierto para un huerto escolar.

- Ubicado en la zona urbana del municipio de Santa Ana.

- Que contara con el respaldo institucional para el fortalecimiento del huerto, en vías a la mejora de la práctica educativa.

En tal sentido, se seleccionó al Centro Escolar Instituto Nacional de Santa Ana (INSA) ${ }^{2}$, específicamente en el sector del Tercer ciclo de educación básica. Dicho centro se encuentra ubicado, específicamente, en la 31 calle poniente y avenida Santa Ana California sur, colonia El Palmar, municipio de Santa Ana, del departamento del mismo nombre, El Salvador. Este centro escolar es una de las instituciones educativas del sector público más complejas; además, posee una amplia población estudiantil. Esta institución ofrece sus servicios desde educación básica, en el turno vespertino, hasta bachillerato.

1. Organización de las Naciones Unidas Para la Agricultura y la Alimentación

2. Dentro del documento, la autora también se referirá a este centro escolar mediante su acrónimo. 
Para implementar la investigación se realizó una revisión del $\mathrm{PEA}^{3}$ de la institución permitió un respaldo de las acciones e iniciativas encaminadas al desarrollo e implementación del huerto escolar como un instrumento educativo. Durante el diagnóstico se encontró que la finalidad del mismo se había limitado a la producción; y se había dejado de lado su utilización como herramienta pedagógica para el desarrollo de contenidos curriculares, debido a la falta de instrumentos que facilitaran y orientaran al docente para utilizarlo dentro de su práctica educativa.

Para retomar las acciones educativas relacionadas con el huerto escolar, se eligió a los alumnos de octavo grado como población de estudio. De los 374 alumnos inscritos en octavo grado durante el año lectivo 2017; y distribuidos en las diez secciones, que comprende el centro escolar, se aplicó la fórmula de establecimiento de la muestra para reducir la cantidad de participantes. Fue así como se trabajó con una muestra total de 164 estudiantes, pertenecientes a cinco secciones, las cuales fueron: A, B, C, D y E.

A su vez, se eligió la asignatura de de Ciencia Salud y Medio Ambiente como contexto de estudio, ya que el desarrollo curricular de los contenidos que posee facilitaban la investigación. Luego, por medio de instrumentos de evaluación (registro anecdótico y listas de cotejo) se determinó la efectividad de las actividades realizadas durante el proceso de enseñanza-aprendizaje, para posteriormente sistematizar la experiencia de la interven- ción y, elaborar una propuesta metodológica para la utilización del huerto escolar como herramienta pedagógica en el desarrollo de contenidos curriculares y ejes transversales del programa de estudio de la asignatura de Ciencias, Salud y Medio Ambiente.

Esta propuesta incluye, además, la integración de otras asignaturas básicas: Matemática, Lenguaje, Estudios Sociales e Inglés; y fue elaborada en conjunto con maestros de las diferentes especialidades, quienes impartían dichas asignaturas en las secciones que fueron tomadas en cuenta dentro del estudio.

\section{Resultados}

Estrategia I: Implementación de la metodología del Aprendizaje Basado en Proyectos (ABP) utilizando el huerto escolar como recurso pedagógico

El proyecto denominado: "El huerto en $1 \mathrm{~m}^{2 \text { " }}$ fue realizado a partir del segundo trimestre de la asignatura de Ciencias, Salud y Medio Ambiente, el nombre del proyecto surgió a raíz del pequeño espacio que se contaba dentro de la institución para el trabajo práctico, y al gran número de estudiantes que integraban el octavo grado en la institución.

Se implementaron 16 cultivos en parcelas de alrededor de $1 \mathrm{a} 7 \mathrm{~m}^{2}$ de extensión, ubicadas en sector correspondiente a las 10 secciones de octavo grado. Del total de cultivos, nueve especies fueron asignadas a los estudiantes de las cinco secciones que fueron tomados como muestra para esta investigación.

3. Entiéndase como Plan Escolar Anual. 
El trabajo en el huerto escolar, como parte de las actividades relacionas directamente con Ciencias Naturales, fue organizado en sus tres etapas, según lo sugerido por Gómez y Quintanilla (2015): planeación, ejecución y evaluación.

La primera etapa, denominada planeación, consistió en tres acciones específicas:

- Formación de comité del huerto. Se seleccionaron a estudiantes que tuvieran interés y afinidad en la asignatura de Ciencias, Salud y Medio Ambiente, y que además mostraran características de responsabilidad, liderazgo, puntualidad, entre otras; para que fueran líderes de sección. Cabe señalar que se eligieron dos líderes por sección (varón y señorita) para que coordinaran y dirigieran el trabajo de sus compañeros, con el apoyo del docente y de colaboradores. A este comité se le sumaron dos maestros, dos estudiantes universitarios de práctica docente, tres padres de familia y directivos del centro escolar.

\section{- Formación de equipos de trabajo en el} aula. Posteriormente, durante el primer trimestre, se formaron grupos de estudiantes (cinco integrantes por grupo), haciendo un total de ocho grupos por sección. Dentro de estos grupos se desarrollarían las actividades investigativas, experimentales y se realizarían los trabajos rotativos dentro del huerto. En cada grupo se asignó un líder, quien se encargó de coordinar el trabajo en equipo, de acuerdo con la temática asignada dentro del proyecto.

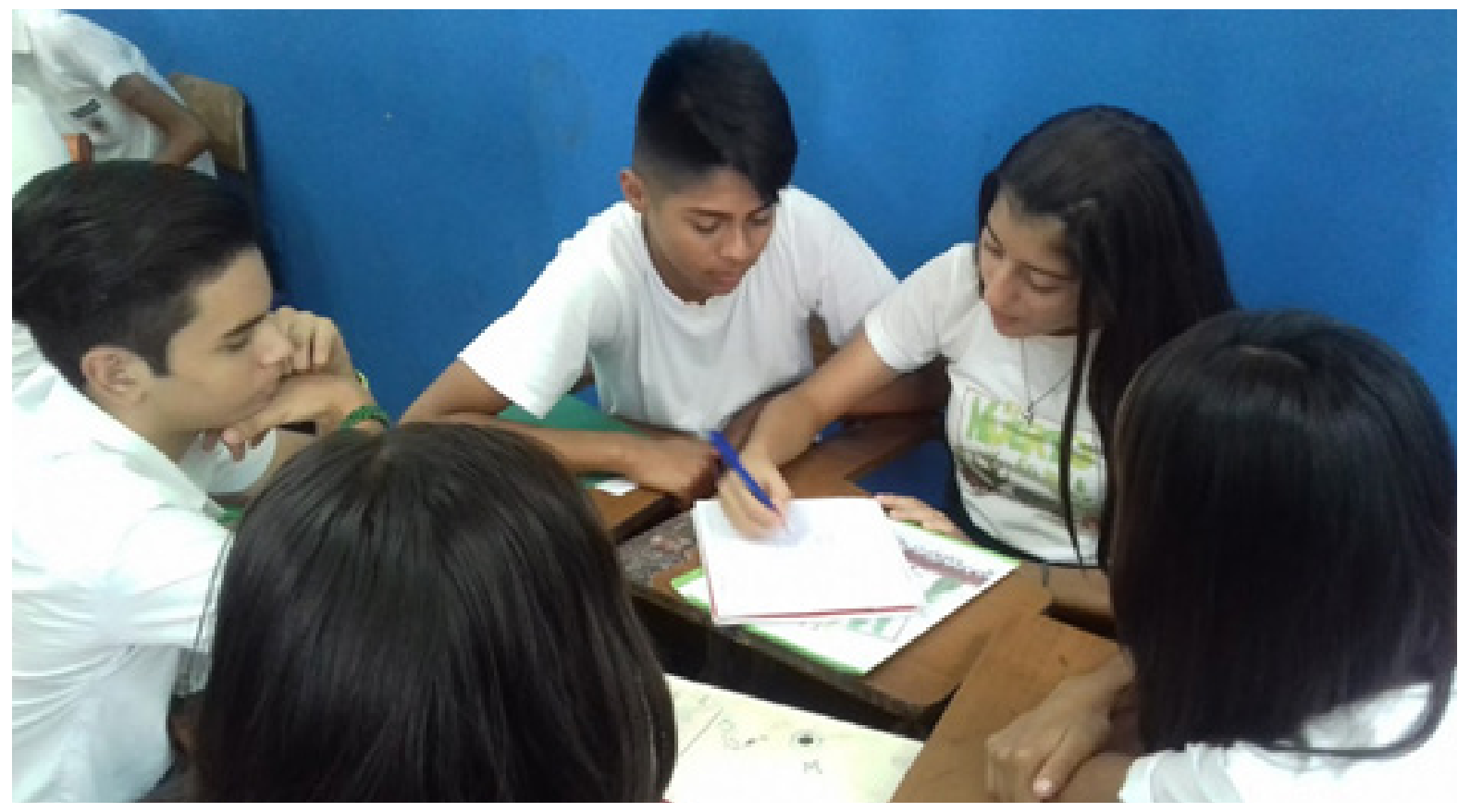

Figura 1. Líder de grupo y sección coordinando las diferentes tareas del proceso investigativo y práctico del huerto. 
- Asignación de especies a cultivar. Se distribuyeron dos especies por sección (comestible, ornamental o medicinal, figura 2) para ser sembradas. A estas se les implementó la metodología del "Huerto en $1 \mathrm{~m}^{2}$, , con prácticas agrícolas tradicionales en vertical (cajas y bolsas) y horizontal (parcelas, siembra en suelo) (ver figura 2). La segunda etapa fue la de ejecución. Aquí se redactaron y ejecutaron dos actividades integradoras durante el segundo $\mathrm{y}$ tercer trimestre. Estas fueron:

\section{a. Trabajemos en "El huerto en $1 \mathrm{~m}^{2 \text { " }}(\mathrm{Se}$ -} gundo trimestre)

Acá se ejecutaron las actividades de implementación del huerto, es decir: limpieza, trabajo en la tierra, siembra, etc., a fin de obtener como producto la implementación exitosa de diferentes métodos de cultivo, así como el conocimiento de actividades agrícolas y caracterización general de las especies a cultivar.

Se evaluó el trabajo cooperativo, la convivencia y el respeto entre compañeros, así como el fomento del liderazgo, la tolerancia y la comunicación asertiva. Esto permitió obtener conocimientos previos sobre las actividades prácticas a realizar (figura 3 ), de acuerdo con las diferentes especies asignadas por sección.

\section{b. Cosechemos en "El huerto en $1 \mathbf{m}^{2 "}$ (Tercer trimestre)}

Durante esta fase se recogió la cosecha, se realizaron actividades de reforestación, mejora de condiciones de jardines y zonas verdes de la institución. Específicamente, como producto de este proyecto se obtuvieron plantas,frutos y semillas de especies reprodu-

\begin{tabular}{|c|c|c|}
\hline \multirow{2}{*}{$\begin{array}{c}\text { Grado y } \\
\text { sección }\end{array}$} & $\begin{array}{c}\text { Especie asignad para } \\
\text { trabajar en huerto } \\
\text { (Nombre científico) }\end{array}$ & $\begin{array}{c}\text { Especie asignada para } \\
\text { trabajar en huerto } \\
\text { (Nombre común) }\end{array}$ \\
\hline \multirow{2}{*}{$\mathbf{8}^{\circ} \mathbf{A}$} & Raphanus sativus & rábano \\
\hline \multirow{2}{*}{$\mathbf{8}^{\circ} \mathbf{B}^{\mathbf{4}}$} & Cucumis sativus & pepino \\
\hline \multirow{2}{*}{$\mathbf{8}^{\circ} \mathbf{C}$} & Spinacia oleracea & espinaca \\
\cline { 2 - 3 } & Apium graveolens & apio \\
\hline \multirow{2}{*}{$\mathbf{8}^{\circ} \mathbf{D}$} & Zea mays & maíz \\
\hline \multirow{2}{*}{$8^{\circ} \mathrm{E}$} & Dianthus caryophyllus \\
\cline { 2 - 3 } & Crotalaria longirostrata & chipilín \\
\hline & Bougainvillea glabra & veranera \\
\hline
\end{tabular}

Figura 2. Especies a investigar y cultivar por parte de los estudiantes.

4. A la sección B solo se le asignó una especie durante los dos trimestres, debido a problemas en la entrega y desarrollo de actividades propuestas durante el desarrollo del proyecto. 
cidas dentro del huerto. También se redactó un documento con el detalle de las acciones realizadas por los estudiantes respecto a los aciertos y desaciertos dentro del trabajo práctico, relacionado con el cuido de la especie dentro del huerto.Al igual que la estrategia anterior, se evaluó la presentación del panel educativo final, así como el trabajo práctico realizado durante el proyecto. (Ver figura 4).

Las actividades realizadas correspondieron a los ocho temas asignados a los grupos de estudiantes por sección. Todo el proceso para la implementación de un huerto se encuentra estipulado en los lineamientos dados por el

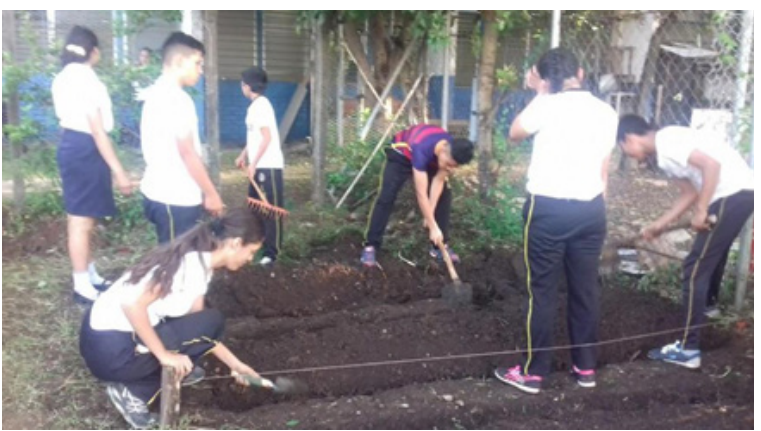

Figura 3. Estudiantes trabajando en la identificación y preparación del suelo del cultivo asignado.

Ministerio de Educación de El Salvador, y que se detallan en el plan de trabajo del proyecto: "Del huerto al emprendimiento" desarrollado para el Centro Escolar INSA (ver figura 5).

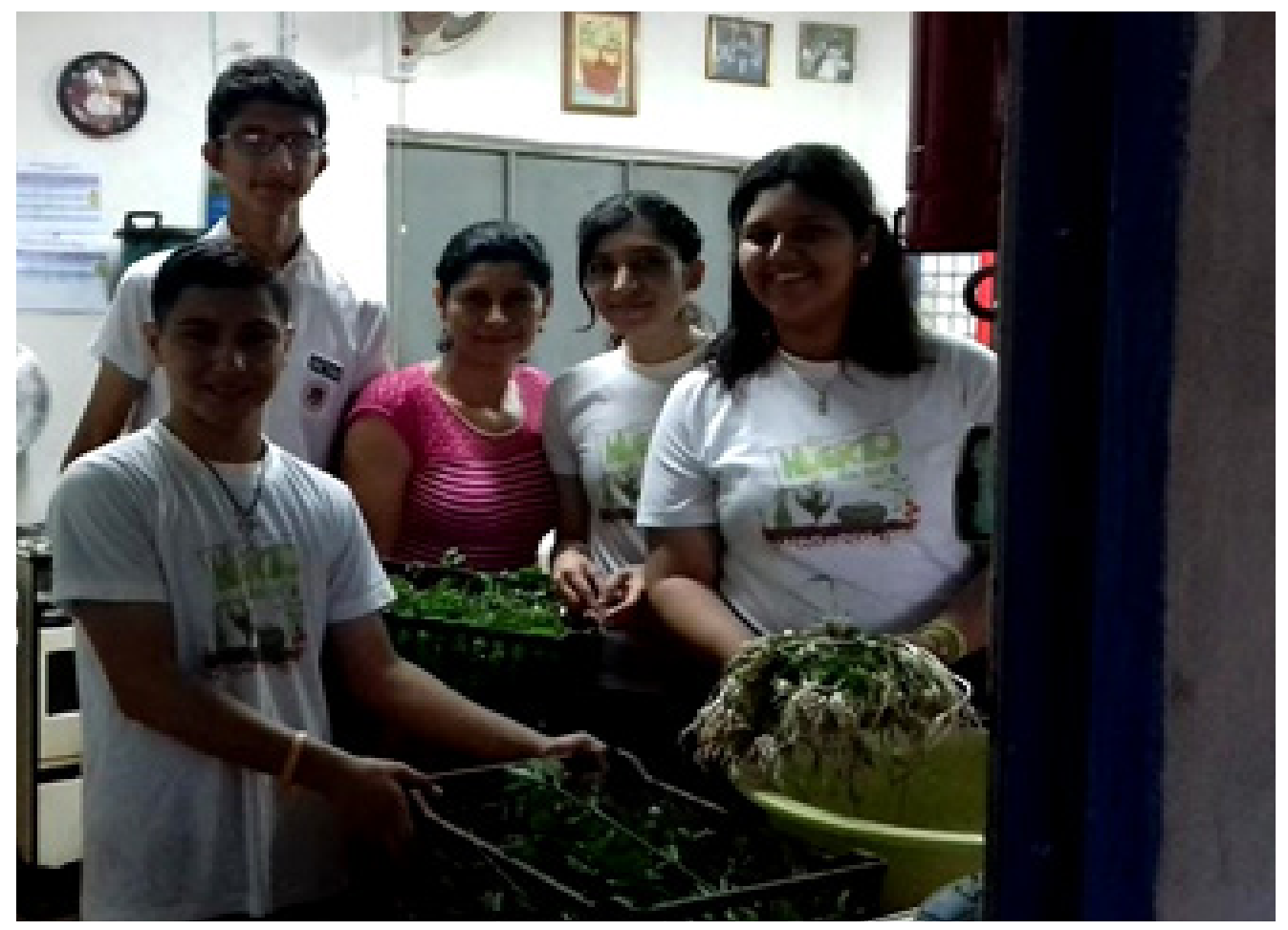

Figura 4. Líderes de sección entregando la cosecha del huerto a la responsable de cocina del centro escolar. 
Desarrollo del trabajo práctico:

- Ejecución de las actividades integradoras: Trabajemos y Cosechemos en "El huerto en $1 \mathrm{~m}^{2}$ "

- Identificación del tipo de suelo

- Preparación de suelo para cultivos

Reproducción de las especies

\section{Instalación de sistemas de riego artesanal}

Taller sobre construcción de compostera:

-Elaboración de abono orgánico compost y utilización de bokashi
La actividad fue realizada con las cinco secciones durante toda la jornada (durante el lapso de las tardes). En las cinco jornadas de clase, la participación fue de doce estudiantes (dos grupos, uno por temática) de cada sección, incluidos los líderes de huerto de la sección.

Los demás estudiantes de la sección trabajaron en avances de la fase de investigación del tema (fase teórica, previa al trabajo práctico). En caso de haber culminado el trabajo práctico, se organizaban y/o trabajaban en el salón de clase para la elaboración de un resumen y un panel educativo.

El trabajo práctico en el huerto fue difícil debido a la carencia de herramientas para las actividades de labranza. Los estudiantes colaboraron con algunas herramientas de sus casas; también hubo padres de familia que se involucraron activamente en el trabajo.

Se trabajó con siete estudiantes (un grupo y dos líderes) en las cinco horas de duración de las clases.

Junto con las cinco secciones se desarrolló la práctica de siembra de especies, a través de mecanismos de reproducción sexual y asexual, así como el establecimiento del semillero.

No se contó con los materiales necesarios para el establecimiento del semillero, por lo que se improvisó con materiales, tales como estantes viejos y gavetas para la siembra en almácigo.

Se instalaron doce sistemas de siembra con la colaboración de padres de familia, utilizando sistemas de riego por aspersión y goteo.

En el desarrollo de la actividad hubo inconvenientes, debido a la falta de una tubería que generara la presión necesaria de agua para el funcionamiento adecuado.

En la práctica fue notoria la fundamentación teórica estudiada, el plan previo (croquis) para su instalación por parcelas y la organización de los equipos; así como el cooperativismo en la compra de los materiales con ayuda de todo el estudiantado de la sección.

Se desarrolló en una sola jornada, en donde participaron aproximadamente 18 estudiantes de las 5 secciones a quienes les correspondía esta temática. Para esto se contó con dos horas clases para su construcción.

Algunos de los maestros de las demás secciones no otorgaron permiso a los estudiantes para participar en la actividad.

No se contaba con las herramientas necesarias para la construcción de la misma, por lo que los estudiantes fueron quienes llevaron las herramientas para facilitar el trabajo práctico. 


\begin{tabular}{|c|c|}
\hline $\begin{array}{l}\text { Charla sobre utilización de } \\
\text { abono orgánico y fertilizante }\end{array}$ & $\begin{array}{l}\text { Esta actividad se realizó con aproximadamente } 35 \text { alumnos de } \\
\text { las cinco secciones, quienes desarrollarían el tema "Utilización } \\
\text { de abono orgánico y fertilizante" y a los líderes de sección como } \\
\text { parte de la actividad integradora dentro de la asignatura Ciencias, } \\
\text { Salud y Medio Ambiente. }\end{array}$ \\
\hline Actividades de mantenimiento & $\begin{array}{l}\text { Estas actividades se desarrollaron posteriormente a la siembra. Fue- } \\
\text { ron hechas por estudiantes tanto dentro de las horas clase como en } \\
\text { el lapso de los recreos. } \\
\text { Al igual que los grupos control de plagas y abono orgánico y ferti- } \\
\text { lizante, los alumnos realizaron un trabajo rotativo para visitar sus } \\
\text { parcelas y aplicar los procedimientos aprendidos en los talleres y en } \\
\text { la fase de investigación. }\end{array}$ \\
\hline $\begin{array}{l}\text { Charla-taller sobre Manejo integrado de } \\
\text { plagas y utilización de insecticida foliar } \\
\text { (práctica de manejo integrado de plagas) }\end{array}$ & $\begin{array}{l}\text { Se realizó con aproximadamente } 35 \text { alumnos de las } 5 \text { secciones, } \\
\text { quienes desarrollarían el tema "Control de plagas en los cultivos" y a } \\
\text { los líderes de sección como parte de la actividad integradora dentro } \\
\text { de la asignatura de Ciencias, Salud y Medio Ambiente. } \\
\text { La actividad permitió que los estudiantes realizaran el control de } \\
\text { plagas en las especies del huerto de forma orgánica, económica y sin } \\
\text { riesgos a su salud. }\end{array}$ \\
\hline Evaluación de panel educativo & $\begin{array}{l}\text { Cada grupo presentó los resultados de todo su proceso de acuerdo } \\
\text { con el tema asignado. } \\
\text { En la primera actividad integradora se presentó la fase investigativa- } \\
\text { documental (planeación de lo que se iba hacer con un fundamen- } \\
\text { to teórico); en la fase práctica (segunda actividad integradora) se } \\
\text { presentaron los resultados, tomando evidencias de todo el proceso; } \\
\text { aciertos y desaciertos, presentando también el resumen por escrito } \\
\text { del tema asignado, el cual debía estar anexado al diario del huerto } \\
\text { junto con los demás temas de los otros grupos. }\end{array}$ \\
\hline $\begin{array}{l}\text { El emprendimiento con } \\
\text { el producto de la cosecha }\end{array}$ & $\begin{array}{l}\text { Esta actividad fue realizada al momento de la presentación del panel } \\
\text { educativo. Lo estudiantes presentaron productos con las plantas y/o } \\
\text { frutos obtenidos en el huerto con fines económicos y medicinales, } \\
\text { estableciendo una sencilla idea de negocio (emprendimiento) con } \\
\text { los resultados obtenidos de la especie asignada. }\end{array}$ \\
\hline Cosechando en el huerto & $\begin{array}{l}\text { Primera cosecha: cilantro, apio y cebollín } \\
\text { Segunda cosecha: rábano, tomate, cilantro y apio. } \\
\text { La producción obtenida permitió variar el menú de la merienda es- } \\
\text { colar a los estudiantes del sector de Tercer ciclo. }\end{array}$ \\
\hline
\end{tabular}

Figura 5. Detalle de las diferentes actividades realizadas durante los dos últimos trimestres del año escolar 2017 en el huerto escolar. 
Finalmente, en la fase de evaluación se utilizaron los instrumentos siguientes:

\section{a. Registro anecdótico: El diario del huerto}

Consistió en un cuadro matriz, el cual tuvo como objetivo registrar las diferentes actividades realizadas por los estudiantes en el grupo (la duración, descripción de la actividad, observaciones), así como un espacio para sello y firma del docente, de acuerdo con la temática asignada. En caso de que no cumplirse con lo acordado, los alumnos perdían su firma y sello, implicando una menor calificación al finalizar el proyecto.

\section{b. Listas de cotejo}

Se elaboraron dos:

La primera fue para la evaluación del panel educativo; el cual reflejaba la evaluación de competencias propias de la asignatura de acuerdo con los contenidos desarrollados, utilizando el huerto como recurso pedagógico. Es decir, valoraba la parte técnica-conceptual del alcance del proyecto, de acuerdo con la asignatura de Ciencias, Salud y Medio Ambiente.

La segunda lista de cotejo se utilizó para la evaluación del diario del huerto, en el cual se evaluó la parte actitudinal, práctica de valores y actitudes ante el trabajo en equipo. Las valoraciones fueron a partir de los criterios: trabajo en equipo, resultados en el huerto, cumplimiento de las normas del huerto, así como el número de sellos ganados durante los trimestres (ver figura 6).

Los contenidos programáticos en la asignatura de Ciencias, Salud y Medio Ambiente y en el proyecto "El Huerto en $1 \mathrm{~m}^{2 \text { ", generó }}$ productos y el desarrollo de competencias determinadas por el currículo nacional, en los alumnos del octavo grado. Fue evidente el alcance de los mismos, gracias a la implementación de herramientas que demostraron el aprendizaje obtenido por los estudiantes durante el año escolar (ver figura 7).

La evaluación arrojó como resultado las calificaciones obtenidas durante el segundo y tercer trimestre de las dos actividades integradoras que formaron parte del proyecto (ver figura 8).

Se observó que los alumnos lograron adaptarse a la estrategia propuesta; sin embargo, la mayoría mostró dificultades en el trabajo en equipo y cooperativo dentro de los grupos de aula. El caso particular fue la sección $B$, quienes mostraron mayores dificultades durante el proceso. Para ello se procedió a realizar cambios dentro de los grupos, a brindar mayor seguimiento y cumplimiento de fechas, con el apoyo de los padres de familia y de la maestra orientadora. Esto con la finalidad de mejorar los hábitos de estudio, la responsabilidad, el compromiso y el trabajo en equipo de la sección.

En esta parte, el acompañamiento, seguimiento y la motivación fueron claves para que los estudiantes desarrollaran el proyecto:

(...) El rol del docente, cuando emplea el aprendizaje cooperativo, es multifacético. Deberá tomar una serie de decisiones antes de abordar la enseñanza, explicarles a los alumnos la tarea de aprendizaje y los procedimientos de cooperación, supervisar el 


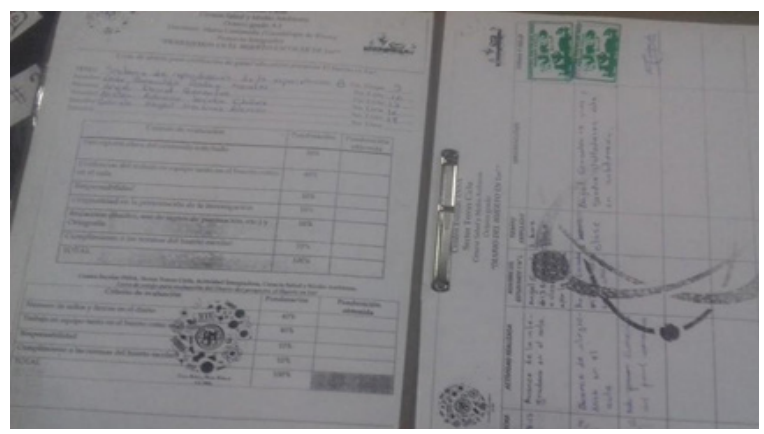

Figura 6. Instrumentos de evaluación utilizados en el proyecto: izquierda, listas de cotejo utilizadas; derecha, registro anecdótico. trabajo de los equipos, evaluar el nivel de aprendizaje de los alumnos y alentarlos a determinar con qué eficacia están funcionando sus grupos de aprendizaje (...) (Johnson, D. W., Johnson, R. T. y Holubec, E. J.; 1999 p. 4): Todo ello fue pieza clave para la obtención de resultados positivos en los estudiantes, lográndose la consolidación de conocimientos teóricos y prácticos en ellos.

Estrategia II: Implementación de prácticas

\begin{tabular}{|c|c|c|c|}
\hline Periodo & $\begin{array}{c}\text { Temática desarrollada } \\
\text { y asignatura }\end{array}$ & $\begin{array}{l}\text { Competencias a } \\
\text { desarrollar }\end{array}$ & $\begin{array}{c}\text { Evidencias de } \\
\text { enseñanza-aprendizaje }\end{array}$ \\
\hline $\begin{array}{c}\text { Segundo y } \\
\text { Tercer } \\
\text { trimestre }\end{array}$ & $\begin{array}{l}\text { - Los compuestos orgá- } \\
\text { nicos e inorgánicos } \\
\text { - El Ph } \\
\text { - Los ecosistema } \\
\text { - Ecología } \\
\text { - La agricultura (prác- } \\
\text { ticas agrícolas) } \\
\text { - El agua } \\
\text { - Seguridad alimentaria }\end{array}$ & $\begin{array}{l}\text { - Aplicación de procedimien- } \\
\text { tos científicos } \\
\text { - Interpretación de la infor- } \\
\text { mación científica } \\
\text { - Desarrollo de ejes transver- } \\
\text { sales: educación ambiental, } \\
\text { educación para la salud y } \\
\text { educación para la igualdad } \\
\text { de oportunidades. }\end{array}$ & $\begin{array}{l}\text { - Guía impresa para el de- } \\
\text { sarrollo del proyecto in- } \\
\text { tegrador correspondien- } \\
\text { te a cada trimestre. } \\
\text { - } \quad \begin{array}{l}\text { Elaboración de resumen y } \\
\text { trabajo grupal en el aula. }\end{array} \\
\text { - } \quad \text { Diario del huerto }\end{array}$ \\
\hline $\begin{array}{l}\text { Tercer } \\
\text { trimestre }\end{array}$ & $\begin{array}{l}\text { - Divulgación científica } \\
\text { - El conocimiento cien- } \\
\text { tífico y sus mecanismos } \\
\text { de divulgación }\end{array}$ & $\begin{array}{l}\text { - Utilización del lenguaje } \\
\text { científico } \\
\text { - Interpretación de la infor- } \\
\text { mación científica } \\
\text { - Desarrollo de ejes trans- } \\
\text { versales: educación am- } \\
\text { biental, educación para la } \\
\text { salud y educación para la } \\
\text { igualdad de oportunidades }\end{array}$ & $\begin{array}{l}\text { - Elaboración de panel educa- } \\
\text { tivo y resumen. } \\
\text { - Bitácora de la producción } \\
\text { del huerto. }\end{array}$ \\
\hline
\end{tabular}

Figura 7. Contenidos programáticos, competencias y ejes transversales de la asignatura de Ciencia, Salud y Medio Ambiente de octavo grado, producto de los aprendizajes del proyecto integrador utilizando el ABP. Fuente: Programa de Estudio de Ciencia Salud y Medio Ambiente para el Tercer ciclo de educación básica (MINEDUCYT, 2009, p.115-117). 
de laboratorio en vivo, utilizando el huerto como recurso pedagógico

Las prácticas de laboratorio en vivo fueron realizadas de acuerdo con la planificación de contenidos en el programa de estudios. Se utilizaron tanto en el huerto como en el salón de clases, al cual se trasladaron organismos vivos (plantas) (ver figura 9).

El desarrollo de las prácticas de laboratorio en vivo fueron una estrategia que ayudó en gran medida a la consolidación de contenidos programáticos, cumpliendo con el alcance de los objetivos propuestos.

Cabe señalar que el criterio 7 , donde se evidenció que los alumnos eran renuentes al trabajo en equipo, pero que a medida fueron siguieron las indicaciones, y con la orientación requerida, estos se adaptaron a las actividades y comenzaron a establecer la comunicción de grupo, tolerancia y respeto; elementos primordiales a considerar para el éxito de las actividades en el aula.
El aprender haciendo, mediante el descubrimiento, fue el factor que captó la atención del alumnado para lograr un buen desempeño en el desarrollo de la práctica, aplicando de forma correcta los pasos del método científico para la resolución de una problemática. (Ver figuras 11 y 12).

Las prácticas de laboratorio en vivo permitieron, además, enlazar lo que fue el huerto escolar y las tecnologías de la información y la comunicación (TIC) en la enseñanza de las Ciencias. Para ello se recurrió a la red social de Facebook, en donde se creó una página para que la docente de Ciencias, Salud y Medio Ambiente brindara orientación a los estudiantes; respondiera consultas sobre los documentos, y compartiera infografías e imágenes que permitieran ampliar el desarrollo de los contenidos conceptuales de la asignatura (ver figura 13).

\section{Promedios finales Actividad Integradora de segundo y tercer trimestre Octavo Grado, secciones de la A hasta la $\mathrm{E}$}

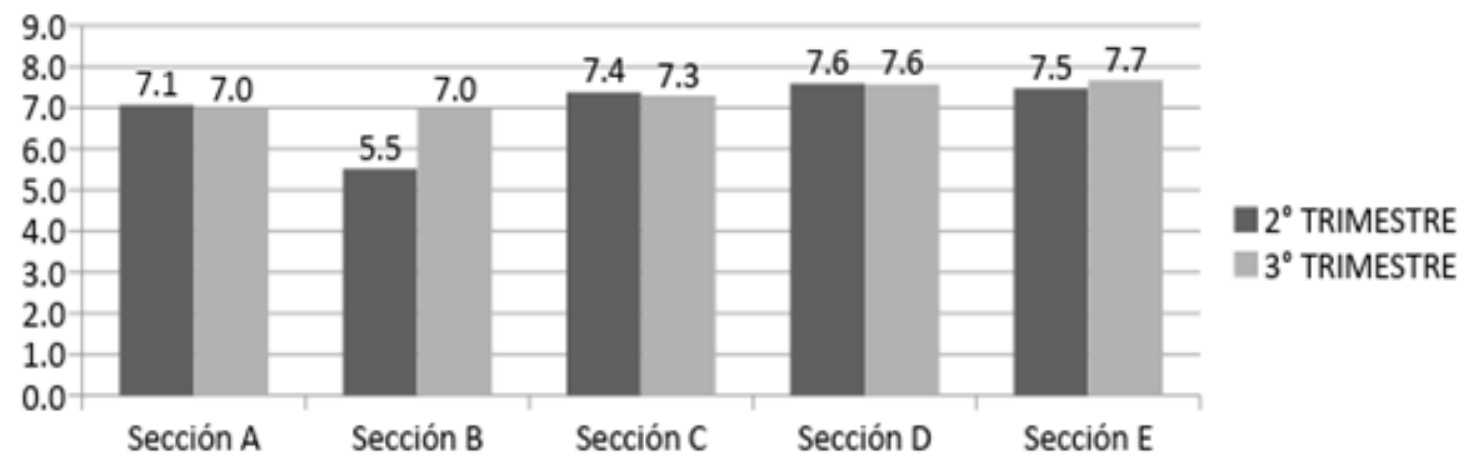

Figura 8. Resultados del proceso de evaluación de las actividades integradoras del primero y segundo trimestre. 


\begin{tabular}{|c|c|c|c|}
\hline Periodo & $\begin{array}{c}\text { Temática desarrollada } \\
\text { y asignatura }\end{array}$ & Competencias a desarrollar & $\begin{array}{c}\text { Evidencias de } \\
\text { enseñanza-aprendizaje }\end{array}$ \\
\hline Tercer trimestre & $\begin{array}{l}\text { - Tipos de tejidos vegetales } \\
\text { - Características de los } \\
\text { organismos vivos: la } \\
\text { absorción y transpira- } \\
\text { ción en plantas } \\
\text { - Los reinos: Reino plantae } \\
\text { - Taxonomía vegetal }\end{array}$ & $\begin{array}{l}\text { - Aplicación de procedimien- } \\
\text { tos científicos } \\
\text { - Interpretación de la informa- } \\
\text { ción científica } \\
\text { - Desarrollo de ejes transver- } \\
\text { sales educación ambiental, } \\
\text { educación para la salud y edu- } \\
\text { cación para la igualdad de } \\
\text { oportunidades. }\end{array}$ & $\begin{array}{l}\text { - Guía resulta de las } \\
\text { prácticas de expe- } \\
\text { rimentación en el } \\
\text { aula/huerto como } \\
\text { laboratorio en vivo }\end{array}$ \\
\hline
\end{tabular}

Figura 9. Contenidos programáticos, competencias y ejes transversales de la asignatura Ciencia, Salud y Medio Ambiente de octavo grado, producto de las prácticas de laboratorio en vivo. Fuente: Programa de Estudio de Ciencia, Salud y Medio Ambiente para el Tercer ciclo de educación básica (MINEDUCYT, 2009, pp.115-117).

El desarrollo de la experimentación en el aula ecológica permitió poner en práctica los conocimientos conceptuales de los estudiantes; además logró mayor motivación e interés de parte de ellos en el proceso (ver figura 10).

\begin{tabular}{|c|c|c|c|}
\hline No. & $\begin{array}{l}\text { Criterios/Nombre de la } \\
\text { práctica experimental }\end{array}$ & $\begin{array}{l}\text { Práctica Experimental 1: Ab- } \\
\text { sorción y transpiración en plan- } \\
\text { tas (22/agosto/17) }\end{array}$ & $\begin{array}{c}\text { Práctica experimental 2: Taxonomía } \\
\text { Vegetal (25/octubre/17) }\end{array}$ \\
\hline 1 & Objetivo de la práctica & $\begin{array}{c}\text { Identificar los procesos de absor- } \\
\text { ción y transpiración del agua en } \\
\text { las especies de plantas cultivadas } \\
\text { en el aula ecológica "El huerto } \\
\text { en } 1 \mathrm{~m}^{2 \prime} \text {, durante la fase de creci- } \\
\text { miento y desarrollo. }\end{array}$ & $\begin{array}{l}\text { Identificar las partes y características } \\
\text { taxonómicas de las plantas que son } \\
\text { parte del vivero en el aula ecológica } \\
\text { "El huerto en } 1 \mathrm{~m}^{2,} \text {, durante la fase de } \\
\text { crecimiento y desarrollo. }\end{array}$ \\
\hline 2 & $\begin{array}{l}\text { Cumplimiento de los } \\
\text { objetivos de aprendizaje }\end{array}$ & Sí se cumplieron & $\begin{array}{l}\text { Sí se cumplió, pero se sugiere reforzar } \\
\text { y mejorar la redacción de la guía para } \\
\text { facilitar el trabajo. }\end{array}$ \\
\hline 3 & $\begin{array}{l}\text { Adecuación de los con- } \\
\text { tenidos de aprendizaje } \\
\text { a abordar }\end{array}$ & $\begin{array}{l}\text { Se adecuaron los contenidos de } \\
\text { las unidades } 5 \text { y } 6 \text { (ver figura 9). }\end{array}$ & $\begin{array}{l}\text { Se adecuaron los contenidos de la uni- } \\
\text { dad } 7 \text { de la asignatura (ver figura 9). }\end{array}$ \\
\hline 4 & $\begin{array}{l}\text { Propiciación de las condi- } \\
\text { ciones para el aprendizaje } \\
\text { esperado }\end{array}$ & $\begin{array}{l}\text { Conveniente, ya que hubo } \\
\text { apoyo por parte de otros agen- } \\
\text { tes al proceso (Alumnos de } \\
\text { Práctica Docente Universitaria } \\
\text { del Profesorado en Biología y } \\
\text { Técnico en Agronomía). }\end{array}$ & $\begin{array}{l}\text { Las condiciones fueron propicias, debi- } \\
\text { do a que hubo apoyo por parte de otros } \\
\text { agentes al proceso (Alumnos de Práctica } \\
\text { Docente Universitaria del Profesorado } \\
\text { en Biología y Técnico en Agronomía). }\end{array}$ \\
\hline
\end{tabular}




\begin{tabular}{|c|c|c|c|} 
No. & $\begin{array}{c}\text { Criterios/Nombre de la } \\
\text { práctica experimental }\end{array}$ & $\begin{array}{c}\text { Práctica Experimental 1: } \\
\text { Absorción y transpiración en } \\
\text { plantas (22/agosto/17) }\end{array}$ & $\begin{array}{c}\text { Práctica experimental 2: Taxo- } \\
\text { nomia }\end{array}$ \\
\hline 5 & $\begin{array}{c}\text { Actitud de interés presenta- } \\
\text { da por el estudiante para la } \\
\text { continuidad de la actividad }\end{array}$ & $\begin{array}{c}\text { La mayoría de estudiantes afir- } \\
\text { mó tener interés en la actividad. } \\
\text { Esto basado en las opiniones } \\
\text { vertidas por los alumnos en la } \\
\text { guía de trabajo. }\end{array}$ & $\begin{array}{c}\text { La mayoría de estudiantes afirmó } \\
\text { tener interés en la actividad, } \\
\text { según las opiniones vertidas por } \\
\text { ellos en la guía de trabajo. }\end{array}$ \\
\hline 6 & $\begin{array}{c}\text { Dominio de los contenidos } \\
\text { de aprendizaje }\end{array}$ & $\begin{array}{c}\text { Demostraron gran dominio } \\
\text { de los contenidos desarro- } \\
\text { llados en clase. }\end{array}$ & $\begin{array}{c}\text { Demostraron gran dominio e } \\
\text { interés durante el desarrollo } \\
\text { de la actividad. }\end{array}$ \\
\hline 7 & $\begin{array}{c}\text { Integración de las partes } \\
\text { (miembros) al trabajo } \\
\text { cooperativo }\end{array}$ & $\begin{array}{c}\text { Dificultades en el seguimiento } \\
\text { de indicaciones falta de ac- } \\
\text { titudes como respeto, respon- } \\
\text { sabilidad y apoyo en el trabajo } \\
\text { (especialmente la sección B). }\end{array}$ & $\begin{array}{c}\text { Se mejoró el trabajo en equipo, la } \\
\text { actitud y, por tanto, se observa- } \\
\text { ron buenos resultados en todo el } \\
\text { trabajo. }\end{array}$ \\
\hline
\end{tabular}

Figura 10. Resultados de las prácticas de laboratorio en vivo 1 y 2 , diseñadas para el proyecto.

\section{Discusión}

La utilización del huerto escolar como herramienta pedagógica, desarrollando la metodología de Aprendizaje Basado en Proyectos y la utilización de prácticas de laboratorio en vivo, resultó ser práctico, factible y exitoso en la enseñanza de la asignatura de Ciencias, Salud y Medio Ambiente, no solo para desarrollar la experimentación, la indagación y la investigación; sino también para formar actitudes de responsabilidad, compromiso, tolerancia y sana convivencia en los estudiantes. Estos elementos son importantes en los contextos en los cuales se encuentran los centros educativos salvadoreños en la actualidad.

El huerto escolar es una herramienta pedagógica que tiene un fuerte componente social, $y$ permite a su vez la interacción de los alumnos con toda la comunidad educativa. Es por ello que el desarrollo de esta investigación no solo favoreció el proceso de enseñanza apren-

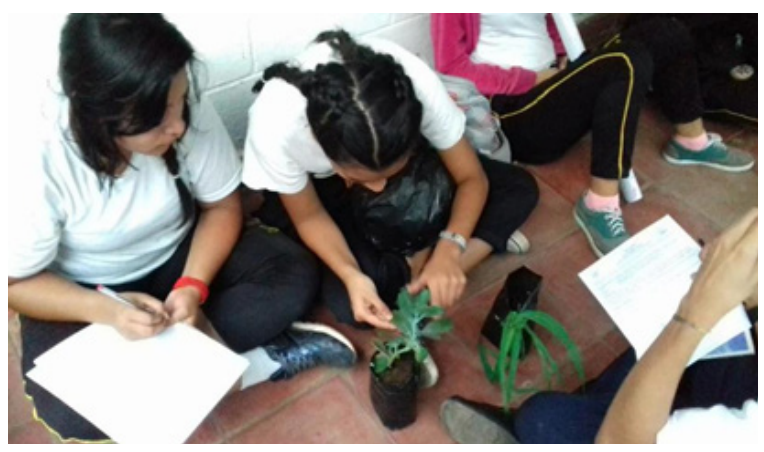

Figura 11. Estudiantes realizando el trabajo práctico experimental

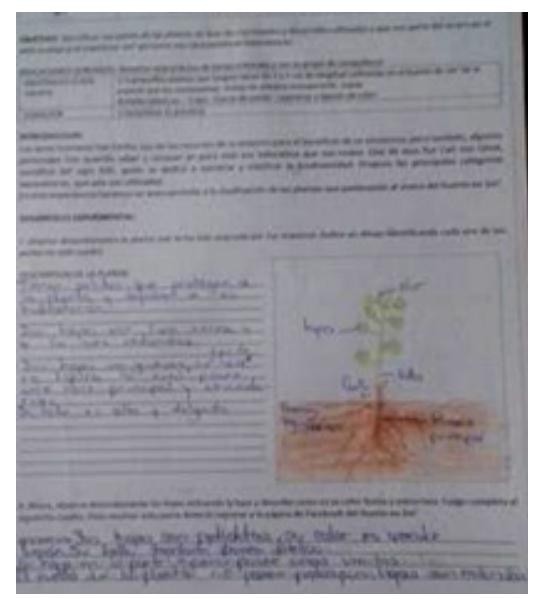

Figura 12. Hoja de práctica experimental que muestra los resultados encontrados por estudiantes. 
dizaje de la asignatura en cuestión, también fue un aporte a la promoción de la sana convivencia y liderazgo dentro de las secciones de octavo grado. Se fomentó el trabajo cooperativo y fortaleció la ejecución del espacio amigable de convivencia y atención preventiva para la niñez, adolescencia y juventudes, desarrollado por el Ministerio de Educación, Ministerio de Salud, Policía Nacional Civil y otras entidades gubernamentales dentro del Centro Escolar INSA.

Los resultados demostraron que, a pesar de que su población estudiantil es compleja, por ser muy numerosa, estar en un espacio reducido y tener pocos recursos disponibles, existe un alto potencial para lograr un huerto escolar; que fomente la utilización de nuevas tecnologías, fortalezca aún más la merienda escolar y desarrolle fuertes competencias tecnológicas-científicas en los estudiantes de tercer ciclo de educación básica.
Además, se incentivó al alumnado a participar en concursos organizados por el Ministerio de Educación, Ciencia y Tecnología de El Salvador, denominados "Arte en mi huerto escolar" (dibujo, pintura y poesía); y a los docentes se les motivó a participar en el concurso "Utilización del huerto como recurso pedagógico". En este último concurso, la institución obtuvo el primer lugar a nivel departamental con el proyecto "El huerto en $1 \mathrm{~m}^{2}$ ".

Con respecto a la primera intervención realizada, correspondiente al trabajo de campo en el huerto, el inconveniente presentado fue la falta de recursos y herramientas que facilitaran el trabajo práctico; además de la apatía por el trabajo en grupo y la puesta en práctica del aprendizaje competitivo y no cooperativo. Esto fue evidente en el caso particular de octavo grado sección B; no obstante, se tomaron las medidas necesarias (cambio en los grupos, delimitación de reglas y normas; establecimiento de fechas límites para la entrega se-

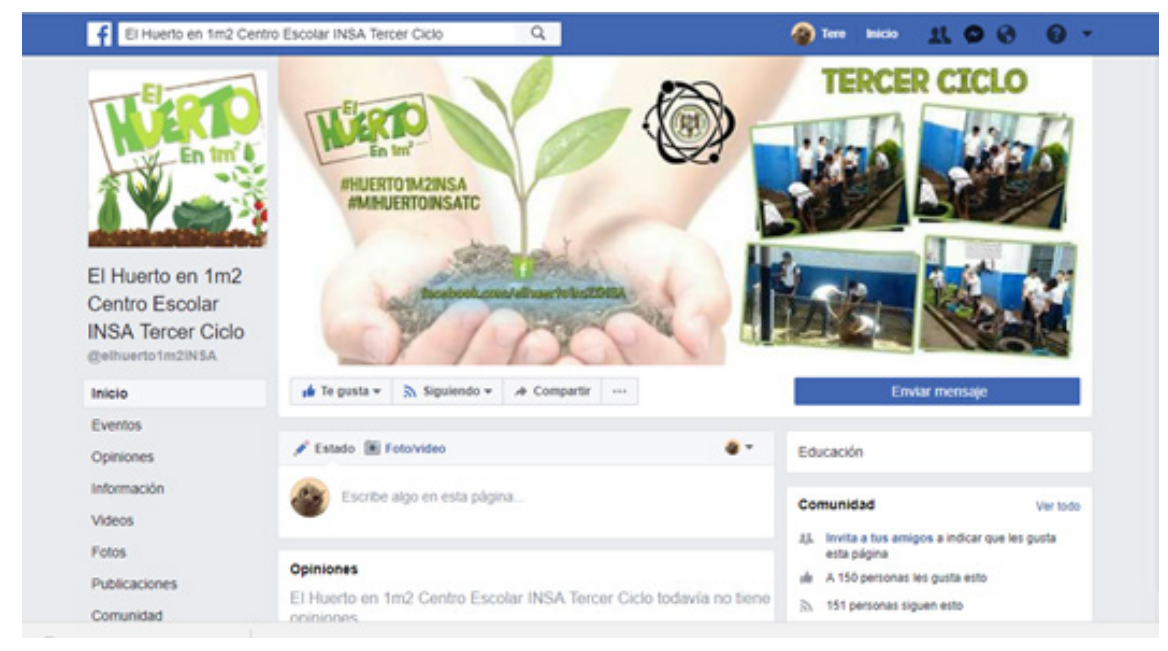

Figura 13. Muestra de la página creada en Facebook para divulgar los resultados educativos de "El huerto en $1 \mathrm{~m}^{2}$ ".

Fuente: https://www.facebook.com/elhuerto1m2INSA/ 
manal o diaria y mayor comunicación entre docente y alumnos) para adaptar la estrategia al grupo de estudiantes. Posterior a la primera evaluación, se observó un cambio actitudinal, un mayor liderazgo y compromiso por parte de los alumnos.

En relación a la segunda intervención, el desarrollo de prácticas de laboratorio en vivo dentro del huerto permitió la contextualización de contenidos, como sugerencias de cambios a la guía metodológica se tomó en cuenta la modificación en la redacción de indicaciones a los estudiantes, quienes tuvieron inconvenientes en la compresión de algunas preguntas formuladas en la guía práctica entregada a cada uno de los grupos.

Con respecto a la utilización de las TIC y el huerto escolar en las practicas experimentales, resultó ser una experiencia interesante para los estudiantes y enriquecedora al docente. Esta actividad permitió compensar la falta de libros que no se encuentran a disposición dentro de la institución, y que motivaron la búsqueda en la web con respecto a las especies y las temáticas abordadas. Los recursos digitales permitieron compartir sugerencias de sitios web confiables e interactivos, donde se encontró información básica para la comprensión del tema asignado o trabajo práctico realizado.

Es posible afirmar que el trabajo por proyectos responde al compromiso social que tiene la enseñanza de la asignatura de Ciencias, Salud y Medio Ambiente en cuanto a formar ciudadanos participativos, que busquen mejorar sus condiciones y su entorno; llevándolos a la toma de decisiones para la acción que refleje a una persona alfabetizada científicamente. El huerto escolar es una herramienta que predispone y brinda las condiciones para el desarrollo de una educación en valores, incorporando la transversalidad y la integración de diferentes contenidos programáticos. Es decir, no fomenta el aislamiento en una sola área del conocimiento, ni tampoco limita a los estudiantes a solo desarrollar competencias netamente científicas, sino que, les ayuda a formarse como seres humanos integrales, que aprenden desde la escuela a respetar su entorno y a convivir con quienes les rodean.

\section{Referencias}

Acedo, M. E. (2009). El huerto escolar ecológico

Aymerich, M. I.; Puig, N. S. y Blanch, M. E. (1999). Fundamentación y diseño de las prácticas escolares de Ciencias Experimentales. Enseñanza de las ciencias: revista de investigación y experiencias didácticas, 17(1), 45-59

Centro Escolar Instituto Nacional de Santa Ana (2017). Plan Educativo Anual 
Gómez, A. A. y Quintanilla, G. M., (2015). La enseñanza de las Ciencias Naturales Basada en Proyectos, Editorial Bellaterra Ltda, Santiago de Chile; 1er Edición

Johnson, D. W.; Johnson, R. T. y Holubec, E. J. (1999). El aprendizaje cooperativo en el aula. Buenos Aires: Paidós

Mancilla, Z. A. M. (2014). Cómo enseñar algunos conceptos de las ciencias naturales a partir de experiencias en la huerta escolar generando competencias científicas escolares

Martí, J. A.; Heydrich, M.; Rojas, M. y Hernández, A. (2010). Aprendizaje basado en proyectos: una experiencia de innovación docente. Revista Universidad EAFIT, 46(158), 11-21

Ministerio de Educación, Ciencia y Tecnología de El Salvador (2009). Programa de estudio Ciencia Salud y Medio Ambiente para Tercer Ciclo de Educación Básica

Ministerio de Educación, Ciencia y Tecnología de El Salvador (2013). Orientaciones básicas sobre Educación Ambiental

Ministerio de Educación, Ciencia y Tecnología de El Salvador (2017). Huerto al emprendimiento para el Centro Escolar INSA, Plan de trabajo 